- GDPs often provide preventive advice in the form of a mini lecture to parents and children, often without visual aids or information to take home.

- GDPs need to think about who they deliver their preventive advice to and reflect upon how they might make it more memorable and effective.

- GDPs need simple evidence-based interventions to offer patients that they believe are unlikely to follow preventive advice.

\title{
Exploring factors that influence general dental practitioners when providing advice to help prevent caries in children
}

\author{
A. G. Threlfall, ${ }^{1}$ C. M. Hunt, ${ }^{2}$ K. M. Milsom, ${ }^{3}$ M. Tickle ${ }^{4}$ and A. S. Blinkhorn ${ }^{5}$
}

\begin{abstract}
Objective To increase understanding about how and to whom general dental practitioners provide preventive advice to reduce caries in young children.

Design Qualitative study using semi-structured interviews.

Setting The North West of England. Interviews took place between March and September 2003.

Subjects and methods Ninety-three general dental practitioners practising within the general dental service were interviewed about the care they provide to young children. The interviews were recorded, transcribed and analysed using a constant comparative method.

Results Children with caries were more likely to be questioned about diet and oral hygiene and if dentists believed parents to be motivated they were more inclined to spend time providing advice. Most dentists seemed to believe that education was the key to preventing caries and gave preventive advice in the form of a short educative talk. There was little use of visual aids or material for parents to take home.

Conclusion Preventive advice is given in an ad hoc way with no formal targeting. Most dentists deliver preventive advice as a short educative talk with no props or additional materials. Use of visual aids, providing materials for parents to take home and greater emphasis on partnership might help improve the impact of advice.
\end{abstract}

\section{INTRODUCTION}

An important role for the general dental service is the prevention of tooth decay in young children. Each day, thousands of parents take their children to a general dental practitioner (GDP) and receive as a matter of routine, advice on diet and tooth

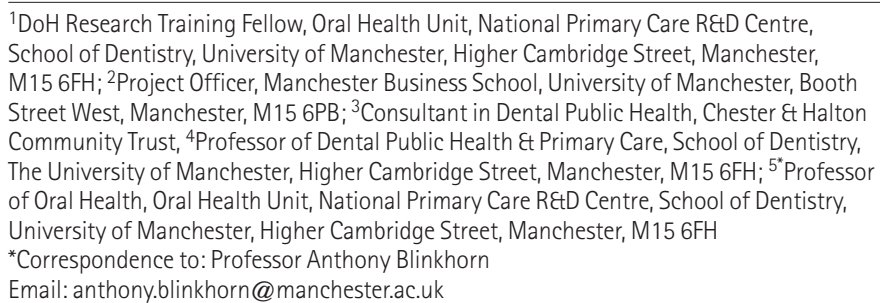

Online article number E10

Refereed paper - accepted 15 December 2005

DOI: $10.1038 /$ bdj.2007.143

(c) British Dental Journal 2007; 202: E10 brushing. In a recent study we interviewed 93 GDPs about the care they provide to children and found that all of them reported giving preventive advice and most covered similar themes, but the content of the advice differed among them in terms of specifics and emphasis. ${ }^{1}$ The impact of a GDP's preventive advice on the oral health of their child patients will be influenced not only by its content, but also by how and to whom it is delivered. In this study we reanalyse the 93 interview transcripts to identify the principle factors that influence individual GDPs' provision of preventive advice. The aim is to increase understanding about how and to whom GDPs provide preventive advice to reduce caries in young children.

\section{MATERIALS AND METHODS}

The method of participant selection and data collection has been previously published. ${ }^{1}$ In brief, GDPs practicing in Lancashire, Cheshire, and Greater Manchester in 2003 were selected at random and sent a letter inviting them to participate. This process continued until approximately 100 dentists had agreed to be interviewed. In total 311 dentists were contacted, 96 agreed to be interviewed of which 3 later declined and the remaining 93 were interviewed. Each participant was interviewed separately by one of three trained interviewers who were not dentists. The interviews took place in the dentists' homes or places of work and were conducted between March 2003 and September 2003. During the interviews each dentist was encouraged to speak freely about the care they provide to the primary dentition. All interviews were tape recorded, numbered for anonymity, and transcribed verbatim.

In this study the data from the 93 transcribed GDP interviews were analysed using a grounded theory approach to identify factors that might influence the provision of preventive advice. This approach is appropriate when there is little already known about the area under investigation. ${ }^{2}$ The constant comparison technique was used to analyse the transcripts. The method used involved initially coding data and constantly comparing new data, firstly with new incidents in the data and then with codes and categories. This was continued until very few or no new categories were emerging from the transcripts. Data analysis was iterative, new emerging codes were used to examine existing codes in more 
depth. The concepts and categories that emerged from the data were formed into themes, which were the key factors that emerged from the transcripts as influencing the provision of preventive advice. $^{2}$ The themes were considered together and discussed in an attempt to identify theory that might connect them. The two researchers (AT and $\mathrm{CH}$ ) undertaking the grounded theory analysis did not have dental backgrounds, which helped ensure theoretical sensitivity, because they did not bring preconceived ideas to the data. Both researchers did, however, believe prior to the analysis that giving advice is a social interaction, likely to be influenced by the beliefs and perceptions of the participants.

\section{FINDINGS}

In the dental surgery the giving of advice to help prevent caries in young children usually involves the GDP, the child, and the adult or adults accompanying the child, which is usually a parent. (The accompanying adult will now be referred to as the parent.) The factors that emerged as influencing the provision of preventive advice are presented by reference to factors associated with the patient, the parent, and factors external to the participants. An overarching theory also emerged, which was that GDPs see themselves as health educators.

\section{Patient factors}

The gender or ethnicity of a child was not important to GDPs when giving preventive advice. Age influenced the delivery of advice as older children were included in the process but it did not appear to influence the likelihood of advice being provided. The attitude and behaviour of a child was reported as being very important for making treatment decisions, but did not emerge as a major factor on the provision of preventive advice. The amount of caries the child had was crucial, however, to both treatment decisions and to the way preventive advice was provided. Children with caries tended to be subjected to questioning about their diet and oral hygiene in an attempt to establish how the caries had occurred, whereas the diet and oral hygiene behaviour of apparently caries-free children tended not to be questioned.

'Why are we seeing this child at three with a hole in its teeth? What is its diet? What does it snack on and how often does it snack?' (1279, male dentist, 34 years experience.)

'Well every case is different I don't have a set spiel that I give to every single child or the mother, but I assess the child's oral hygiene standards and the state of their mouth, their attitude, the attitude of the mother and we go from there. I mean if the child is grossly carious, then we try and find out what they are doing regarding their diet and what not.' (1233, male dentist, 23 years experience.)

'If I see a child and oral hygiene is good, I would say very little about what they are doing because whatever they are doing they are doing alright.' (1150, male dentist, 20 years experience.)

Assessment of the child's oral health also influenced the likelihood of some dentists prescribing or applying fluoride. Children presenting with caries on more than one occasion were either given similar preventive messages again, or were given fluoride tablets or fluoride varnish, but in some cases dentists admitted becoming disillusioned and unmotivated about providing preventive advice because they felt it was not being heeded:

'If I give tablets it's usually for a patient who keeps coming back and back, and you are getting nowhere with the diet advice and the oral hygiene advice...then I am more likely to give fluoride tablets at that stage. But I wouldn't do initially.' (238, female dentist, 5 years experience.)

'The worst cases are when you have been talking to them, and they come back in six months, and they will come back eating a chocolate bar or drinking a can of coke, and you feel like banging your head against the wall thinking why bother.' (367, male dentist 4 years experience.)

\section{Parent Factors}

The GDPs' perception of the accompanying parent, especially their beliefs about parental attitude and motivation, were crucial to the provision of preventive advice. In general, if dentists believed that the parents were well motivated then they were more inclined to spend more time providing preventive advice. A number of participants also made reference to a link between social class and parental motivation about oral health.

'If the parents are well motivated and the kids are well motivated then you can spend time doing a good job on them' (31, male dentist 17 years experience.)

'My philosophy hasn't basically changed. It has changed in that the different practices I have worked in there has been higher or lower motivation. In this practice most of the patients are middle class and they are all motivated.' (196, female dentist 20 years experience.)

'Some mothers, particularly middle class will come in and talk at great length about fluoride... at the other end of the spectrum mothers are stuffing sweets into kids mouths all the time just to placate them' (532, male dentist 25 years experience.)

Linked closely to parental motivation was the notion of success. The dentist's belief that the advice they provided was acted upon by some parents was an important factor in ensuring that they continued to provide advice. Surprisingly when asked directly few dentists could provide specific examples of success.

'Yes I do, and I think it works as long as the parents are willing to listen and do what you say... but I say with 90\% of parents and children it does help to stress prevention.' (2213, female dentist, 16 years experience.)

'I would say I have 50\% success.' (359, male dentist, 13 years experience.)

'The children that come in with extensive caries, we try to educate in a similar fashion, but it tends to be more some battles you win and some you lose. A lot of the parents don't take on board or care about why the problem is there. ... We do win some children and parents over but a lot of them we don't as there is not that much enthusiasm for dental care in the household.' (748, male dentist, 30 years experience.)

The dentist's perception of the level of ignorance a parent had about diet and oral hygiene influenced the advice provided. Dentists reported that many parents were ignorant about the causes of tooth decay and they often tried to make sure that parents understood the causes of decay and the harmful effects of sugar.

'There are a lot of things that they are not aware of, like they don't realise that there is sugar in a lot of drink, and it does pay off to go through all those things.' (2213, female dentist, 16 years experience.)

'I said there is loads of sugar in ice cream and she said (mother) I can't believe that.'(280, male dentist, 21 years experience.)

'I think knowledge, mum didn't really know why the child's teeth were decaying and I think once I explained it the light switched on for mum.' (1013, male dentist 6 years experience.) 


\section{External factors}

Practices with a hygienist tended to have an increased emphasis on dietary advice and oral hygiene instruction and GDPs working with a hygienist often delegated the provision of preventive care to them.

'All children to see a hygienist on a regular basis... because they do the best prevention care' (31, male dentist, 17 years experience.)

'It's the nurses that do that (preventive advice) not me' (1491, male dentist, 19 years experience.)

'Very often I will refer to the hygienist for this kind of instruction as well. Obviously they have more time and they are probably better than me at this kind of thing anyway.' (2440, male dentist, 35 years experience.)

Time was an important factor which participants felt affected their provision of advice. Indeed, the majority of respondents referred to the problem of time restrictions and many linked this problem to the fee structure. Some of the GDPs reported that pressure on their time meant they were unable to provide as much preventive advice as they would have wanted:

'But it's like all the dentistry at the moment I'm afraid, if it's time consuming which it can be, then dentists can be tempted not to do it.' (392, female dentist 26 years experience.)

'I think the major problem for a general dental practitioner is the financial reward for what we are doing. If you get a fixed hourly rate for treating a child patient then you don't have to worry so much about the income that you are getting in by looking after the patient, because prevention does take a lot of time and effort,...' (576, male dentist, 15 years experience.)

'Ideally I would like to spend half an hour with a child, but I don't, it is more likely to be fifteen minutes or twenty minutes.' (802, male dentist, 17 years experience.)

'I think if remuneration was improved, and we got a lot more time, and then get paid for your time to sit them down and talk to mum and dad about dietary advice, and then I would think it would save the Health Service money in the future.' (797, male dentist, 11 years experience.)

An overarching theory emerged from the transcripts; GDPs see themselves in the role of health educators when considering prevention. There was an almost universal belief that caries could be prevented if parents listened to and understood the diet advice and oral hygiene instruction provided. This belief in education as the route to behavioural change seemed to underpin much of the way that advice was given. The majority of dentists relied on verbal advice in the form of a short educative talk and some also handed out leaflets. GDPs often appeared to be unsure about what to do if their educative talks were not working. Diet sheets were tried by a few dentists but those who did try these reported that they were unsuccessful. Although dentists saw themselves as health educators, there was little evidence that they used techniques such as visual aids to increase the impact of their preventive advice.

'The overall aspect for me for children is education and prevention. So we are always looking at here to try and educate both the child and the parent and we are not concerned with where they are at the moment, it is where they are going to go.' (993, male dentist, 2 years experience.)

'I tried doing diet sheets. I gave them diet sheets and asked them to fill them in for two weeks. I tried on about ten or eleven, and none of them bothered, so I stopped doing that.' (367, male dentist 4 years experience.)

'We try to educate the ones that don't look after their kids, but I don't think the compliance is terribly good. There is no evidence of that, but I just feel that, I don't know, I mean you will see the kid again twelve months later and they have got more cavities and their teeth are still covered in plaque. So you know damn well that they have done nothing about it.' (331, male dentist 16 years experience.)

'It's difficult because we have not got many methods or tools to explain things. We have not got models to explain to them this is how you brush your teeth. We haven't got toothbrushes to show them how to clean their teeth or whatever; we do try to get them to bring their own toothbrush in, which helps sometimes.' (367, male dentist, 4 years experience.)

\section{DISCUSSION}

When analysing the transcripts we divided the factors that influence the provision of preventive care into those relating to the patient, the parent and those external to the participants. The most important patient factor was the level of caries. Children with caries were more likely to receive preventive advice and to be questioned about oral hygiene and diet. This confirms the finding from an earlier case notes study, and indicates that many dentists often target preventive care on those children that already have caries. ${ }^{3}$ The dentist's perception about the motivation and attitude of the parent towards oral health influenced both how and to whom the advice was given. In general the dentists were more inclined to give advice and spend more time advising middle class parents, whom they perceived as being more motivated than parents from a lower social class. Pressure on time also emerged as being an important factor, as many dentists reported that they would provide more preventive advice if they were not so busy. Time pressure was inexorably linked to the fee system, with dentists indicating that the current method of payment for children discouraged lengthy sessions of preventive advice. The structure of the practice and in particular the presence of a hygienist also influenced how preventive advice was delivered. Dentists with hygienists tended to delegate much of the preventive advice to the hygienist. In addition, the almost universal belief among the participants that education of patients and parents was crucial to preventing caries fundamentally influenced the whole process.

None of the GDPs in this study reported using a set structure for choosing children to receive preventive advice. Instead, all provided advice in an ad hoc manner based on their own experiences, the level of caries the child had, and their beliefs about the motivation of parents, which tend to be linked to socio-economic class. In this way GDPs informally target advice in two ways, firstly according to caries levels and secondly according to beliefs about motivation. Many participants stated that patients from lower socio-economic groups tended to be less motivated about preventing caries. This observation might be true, but focussing consciously or unconsciously on motivated middle class families will only serve to reinforce the inverse care law. ${ }^{4}$

Delivering preventive advice to people who appear to be unmotivated is likely to be difficult and dentists reported that they became disillusioned when people did not listen or obviously had not acted upon their advice. Some dentists gave fluoride supplements when they felt advice was not working and some repeated their advice even though they thought it would not work. This highlights a key problem; GDPs are uncertain about what to do when their usual delivery of advice is not working. There is a clear need to develop an armoury of simple interventions that GDPs can 
use as a supplement to preventive advice and to try with unmotivated parents.

For many dentists, pressure on time was a key factor in delivering preventive advice. Some dentists would like to talk at length to the families of some patients but felt they could not do this, either because other patients were waiting or because they felt that giving lengthy preventive advice was not a cost-efficient use of their time. Not surprisingly, dentists with a hygienist delegated some responsibility for the provision of preventive advice. This may be a more cost effective method of delivering advice and many of the dentists reported that hygienists are better at delivering preventive advice. However, no trial comparing the effectiveness of advice provided by hygienists and GDPs has been published and for now, in our opinion, it may be best if GDPs provide the key preventive messages and hygienists reinforce these messages by working in partnership with patients to provide practical examples of how to put the advice in to practice. In this way, prevention would remain a core activity of GDPs. It would be unfortunate if it were seen as being a peripheral activity to be delegated.

Almost all the GDPs in this study believed that the key to preventing caries in young children was education and the majority provide preventive advice verbally, in the form of a mini lecture. This was usually delivered without any visual aids or props and only a minority of respondents stated that they provide diet sheets, leaflets and website addresses. There was a lack of imagination in the delivery of preventive advice and a lack of additional materials for parents to take home, although studies have demonstrated methods specific to young children such as games can help reinforce dental health messages. ${ }^{5}$ Most GDPs seemed to limit their role to being prescriptive, many seeming to model themselves on a teacher in a classroom with parents and patients as their pupils, some of whom were good, and listened attentively, and others of whom were bad and did not listen. There was little evidence among the GDPs of reflection about the way they delivered preventive advice. Few seemed to have reflected on ways to make their preventive advice more memorable or on alternative approaches to learning, for example, working in partnership with parents and children.

'The longstanding criticisms of current oral health promotion practice - that it is professionally dominated, individualistic, behaviourally orientated and prescriptive - still retain their valid- ity when current practice and examples of innovation are reviewed.' These words, published in 1993, remain accurate over ten years later. ${ }^{6}$ The arrival of the new dental contract provides an opportunity for change by placing prevention at the heart of dental care and allowing dentists to spend more time with children. This opportunity will be lost unless efforts are made to both improve the content and delivery of preventive advice and to uncover simple interventions that might result in improving usage of fluoride toothpaste and changing children's diets. These interventions will need to be developed in partnership with patients if the prescriptive mindset of GDPs towards prevention is to be challenged. Research can be undertaken to test innovative approaches and identify better ways of delivering preventive care. Training can be provided, both as part of the undergraduate curriculum and as part of continuing professional development, to improve the delivery of preventive care by promoting a better understanding about counselling skills and educative techniques. In addition, individual GDPs need to reflect on their own delivery of preventive care to identify ways in which it might be improved.

\section{CONCLUSION}

Preventive advice provided in the dental practice is given in an ad hoc way with no formal targeting of patients. Most GDPs tend to deliver preventive advice in a similar manner, a short educative talk with no props or additional materials. In addition, there was no planned reinforcement of advice. Greater use of visual aids, providing materials for parents to take home, and greater emphasis on partnership might help improve the impact of GDPs' advice.

1. Threlfall A G, Milsom K, Hunt C, Tickle M, Blinkhorn A S. Exploring the content of the advice provided by general dental practitioners to help prevent caries in young children. Br Dent J 2007: 202: E9.

2. Strauss A, Corbin J. Basics of qualitative research: grounded theory procedures and techniques. Newbury Park: Sage, 1990.

3. Tickle $M$, Milsom K M, King D, Blinkhorn A S. The influences on preventive care provided to children who frequently attend the UK general dental service. BrDent J 2003; 194: 329-332.

4. Tudor Hart J. The inverse care law. Lancet 1971; 1: 405-412.

5. Makuch A, Reschke K. Playing games in promoting childhood dental health. Patient Educ Couns 2001; 43: 105-110.

6. Croucher R. General dental practice, health education, and health promotion: a critical appraisal. In Schou L, Blinkhorn A S (eds) Oral Health Promotion. pp 164. Oxford: Oxford University Press, 1993. 